L. A. Willson

Erwin W. Fick Observatory

Physics Department

lowa State University

Mira variables are found at the tip of the asymptotic giant branch, with $\mathrm{L} \approx 3000-5000 \mathrm{~L}_{6}$ and $\mathrm{T}_{\mathrm{e}} \approx 3000 \mathrm{~K}$. (Feast 1981; Willson 1981a). They are fundamental mode pulsators (Willson 1979, 1981a). A typical Mira has $\mathrm{P} \sim 350$ days, $\mathrm{R} \sim 200-300 \mathrm{R}_{\Theta}, \mathrm{M} \sim 1-2 \mathrm{M}_{\odot}$ (Willson $1979 ; 1981 \mathrm{a}$ ). From the atmospheric velocities of the Miras plus a fundamental mode period-massradius relation one finds present masses for the Miras which are not very different from their progenitor masses (Willson 1981a). This suggests that pre-Mira mass loss is moderate -- $\lesssim 20 \%$ of the mass is lost before pulsation starts. In fact one expects only moderate mass loss before the Mira stage; using for example Reimers (1975) formula

$$
M=\eta 410^{-13} \mathrm{LR} / \mathrm{M} \quad \mathrm{M}_{6} / \mathrm{yr}
$$

with presently favored values of $\eta\left(\frac{1}{4} \leqslant \eta \leqslant \frac{1}{2}\right.$ e.g. Renzini 1981) yields a terminal pre-Mira mass loss rate $\sim 10^{-7} \mathrm{M}_{\odot} / \mathrm{yr}$. Since $\mathrm{L} \sim \mathrm{e}^{\mathrm{t} / \mathrm{t}}$ ev the total mass lost is approximately $\dot{M}_{f} t_{e v}$; with $t_{e v} \leqslant 10^{6}$ yrs (Paczynski 1974; Wood \& Cahn 1977) $\triangle \mathrm{M} \leq .1 \mathrm{M}_{\odot}$.

Mass loss rates for pulsating stars are expected to be larger than for non-pulsating stars of similar L,R,M, due to either a) Pulsation driving $\dot{\mathrm{M}}$ and/or $\mathrm{b}$ ) pulsation increasing the scale height and thereby enhancing it by other mechanisms. (Willson \& Hill 1979; Wood 1979)

Observed mass loss rates for Mira variables are considerably larger than given by Eq. (1) with $n<1$ : from infrared excesses Gehrz \& Woolf (1971) found typical Mira mass loss rates $\approx 210^{-6} \mathrm{M}_{\odot} / \mathrm{yr}$, with individual stars ranging up to a few times $10^{-5} \mathrm{M}_{\odot} / \mathrm{yr}$. For mass loss rates in excess of $10^{-6} \mathrm{M}_{\odot}$ /year one expects period changes which should be observable, given observations over a sufficiently long time:

$$
\frac{\dot{\mathrm{P}}}{\mathrm{P}}=c_{1} \frac{\dot{\mathrm{M}}}{\mathrm{M}}+c_{2} \frac{\dot{\mathrm{L}}}{\mathrm{L}}=1.5 \frac{\dot{\mathrm{M}}}{\mathrm{M}}+\left(<310^{-7}\right) \mathrm{yr}^{-1}
$$


where $c_{1}, c_{2}$ depend slightly on the PMR relation used and on the evolutionary track ( $\mathrm{T}_{\mathrm{e}} \mathrm{vs}$. $\mathrm{L}, \mathrm{M}$ ) assumed. For o Cet, 360 years of observations give $\dot{M} / M^{2} \leq 2 \cdot 10^{-6} \mathrm{yr}^{-1}$; for $\chi$ Cyg, 290 yrs. give $\dot{M} / M=$ $10^{-5} \mathrm{yr}^{-1}$ (Willson 1981b). Hence observed period changes are consistent with $\dot{\mathrm{M}} \leqslant 10^{-5} \mathrm{M}_{\Theta} / \mathrm{yr}$ for most Miras.

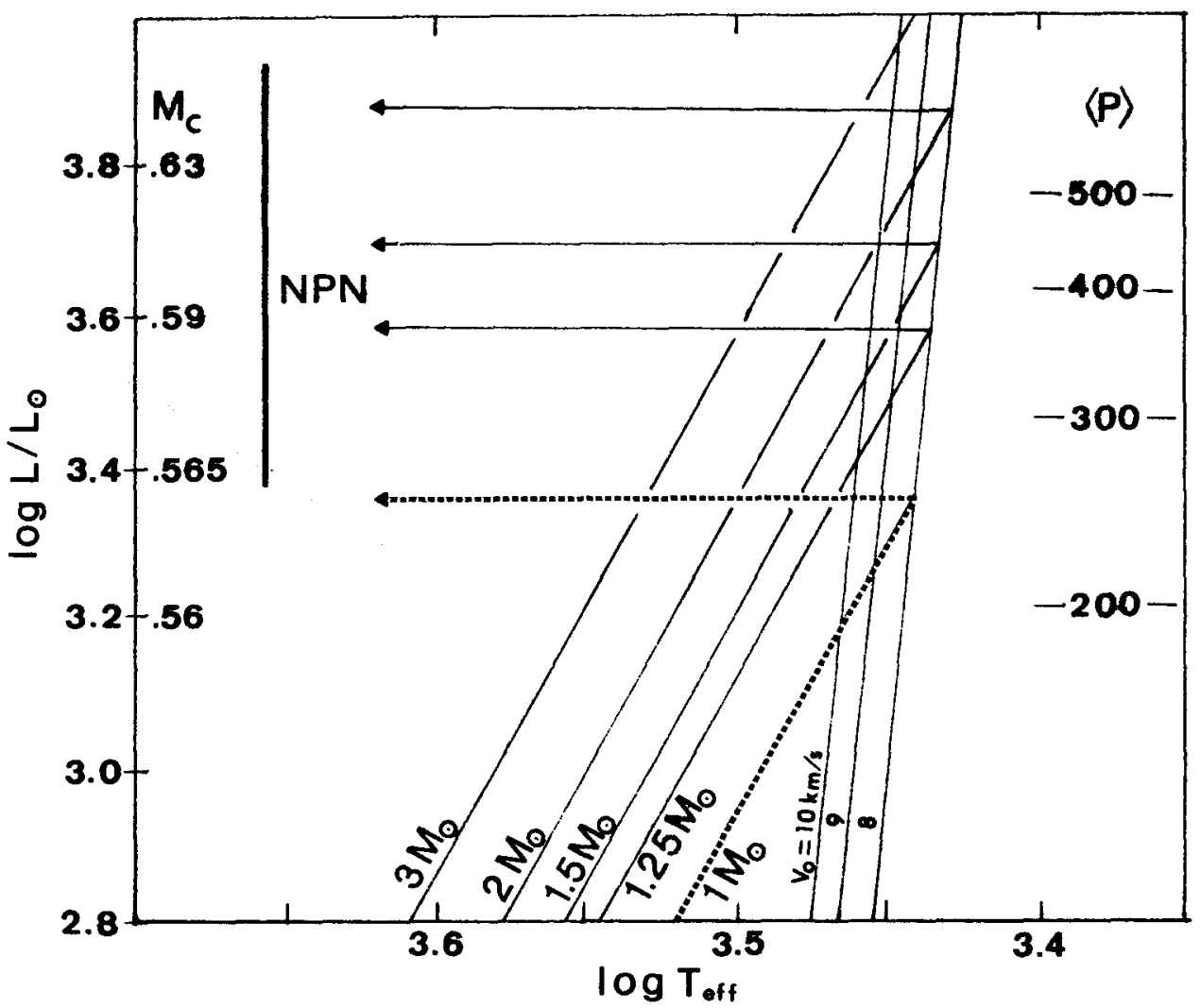

Figure 1. Schematic evolution on the AGB for stars of solar abundance. The Mira strip is defined roughly by $v_{O}=8-10 \mathrm{~km} / \mathrm{s}$ as shown; mass loss during the Mira stage is large, leading to rapid evolution to the left upon depletion of the envelope mass. Mean luminosities for P-200500 days from Feast (1981) are indicated, as are the planetary nebula nuclei of Schonberner \& Weidemann (1980) (NPN). For equations and parameters used see Willson (1981a). 


\section{RESULTS AND CONCLUSIONS}

The resulting evolutionary picture is summarized in Figure 1. Stars evolve up the AGB with only moderate mass loss; at $\mathrm{T}_{\mathrm{e}} \approx 3000 \mathrm{~K}$ Mira pulsation commences, driving the mass loss rate up by at least a factor of 10 and thus removing the entire envelope in a few times $10^{5}$ years. This process leaves a stellar remnant $\approx 0.6 \mathrm{M}_{\odot}$ and a planetary nebula of $\lesssim .5 \mathrm{M}_{\Theta}$, consisting of the last $10^{5}$ yrs of the Mira wind swept up by a more rapid, hotter NPN wind (Kwok, Purton, \& Fitzgerald 1978). Additional support for this picture comes from the agreement between the new Mira luminosities (Feast 1981) and the new masses for the nuclei of planetary nebulae (Schonberner \& Weidemann 1980).

Wood \& Cahn (1977) counted $245 \mathrm{Miras} / \mathrm{kpc}^{3}$; they also derived $\mathrm{dn} / \mathrm{dt}_{\mathrm{pN}} \approx \mathrm{dn} / \mathrm{dt} \mathrm{t}_{\mathrm{MS}}$ for main sequence stars with $\mathrm{M}<\mathrm{M}_{\mathrm{C}}, \mathrm{M}_{\mathrm{C}} \approx 3 \mathrm{M}_{\odot}$. The timescales for Mira evolution with rapid mass loss are nearly 10 times shorter than the Wood \& Cahn time-scales; hence we find $\mathrm{dn} / \mathrm{dt}_{\text {Miras }} \approx$ $10 \mathrm{dn} / \mathrm{dt}_{W C}$ and hence

$$
\mathrm{dn} / \mathrm{dt}_{\mathrm{MS}} \approx \mathrm{dn} / \mathrm{dt}_{\text {Miras }} \approx \mathrm{dn} / \mathrm{dt}_{\mathrm{PN}} \approx 310^{-3} \mathrm{yr}^{-1} \mathrm{kpc}^{-3}
$$

Thus: all low mass stars pass through a stage of Mira variability preceding the planetary nebula stage, and Mira variability appears to be the means by which most stars rid themselves of excess mass to become white dwarfs.

\section{REFERENCES}

Feast, M. 1981, in "Physical Processes in Red Gaints", ed. I. Iben Jr. and A. Renzini, Réide1, Dordrecht, p. 193.

Gehrz, R.D. and Woolf, N. 1971, Ap.J. 165, p. 285.

Kwok, S., Purton, C.R., Fitzgerald, P.M. 1978, Ap.J. (Letters) 219, L125.

Paczynski, B. 1974, "Late Stages of Stellar Evolution" Tayler (Ed) p. 62.

Reimers, D. 1975, "Problems in Stellar Atmospheres and Envelopes"

Ed. Baschek, Kegel, and Traving. Berlin p. 229.

Renzini, A. 1981, (Present volume).

Schonberner, D. and Weidemann, V. 1980, (Preprint; see Weidemann, present volume).

Willson, L.A. 1979, IAU Colloquium \#46.

Willson, L.A. 1981a, (In preparation; short version in Erice conference on "Physical Processes in Red Giants").

Willson, L.A. 1981b, (In preparation--more complete version of present paper).

Willson, L.A. and Hil1, S.J. 1979, Ap.J. 228, p. 854.

Wood, P. 1979, Ap.J. 227, p. 220.

Wood, P. and Cahn, J. 1977, Ap.J. 211, p. 499. 


\section{DISCUSSION}

RUMPL: Could you tell us what drives the mass 1033 from the Mira's?

WILSON: Referring to Willson \& Hill (Ap. J. 228, 854, 1979): a) Lower atmosphere isothermal shocks enhance $H$ but do not suffice to drive $\dot{\mathrm{M}}$; b) As the density decreases the cooling becomes such less efficient, so the shocks become non isothermal at $\sim 2 \mathrm{R}_{*}$ thus substantial $\dot{\mathrm{M}}$ at low $v ; c)$ Around $d \geqslant 5 R_{*}$ dust forms and this accelerates the flow to $v_{\infty}$ $.10 \mathrm{~km} / \mathrm{s}$. Note that all these regions are now observed: a) in the infrared*; b) infrared $*$ and SiO masers*; c) OH masers*\%. *Hinkle, Hall, Ridgway (preprint), **Elitzur, review at Erice.

CARRASCO: For how long a time do you expect a Mira variable to exist? If you consider that the stellar evolution pace is set by conditions of the stellar core but pulsation is set by conditions in the envelope-atmosphere.

WILLSON: The mass loss timescale for $\dot{M}=10^{-6} \mathrm{M}_{\odot} / \mathrm{yr}$ is $\leqslant$ the evolutionary timescale so the star lasts approximately

$$
t=\mathrm{M}_{\text {envelope }} / \dot{\mathrm{M}} \simeq \text { few times } 10^{5} \mathrm{yrs} \text {. }
$$

During this time the luminosity probably only changes by $\log \mathrm{L} \approx 0.1$.

WOOD: 1) one should be very cautious in using rates of period change to determine $\dot{M}$ in Miras, since the luminosities of these stars vary on many timescales due to Helium shell flashing (see Wood and Zarro 1980. Ap. J. in press). 2) You use Feast's luminosities (which are 0.75 mag rainter than previous values) yet you don't use his Teff values (which are $\sim 400 \mathrm{k}$ cooler than the values you selected). Why?

WILLSON: 1) Agreed. I merely pointed out that $\dot{M}=10^{-5} \mathrm{M}_{\odot} / \mathrm{yr}$ is consistent with the appearance of the O-C for $\chi$ Cyg. That doesn't prove that the curvature is due to $\dot{\mathrm{M}}$. We need better $\dot{\mathrm{M}}$ rates. I should point out that my evolutionary scenario used the best observed rates; $\left(\geqslant 10^{-6} \mathrm{M}_{\odot} / \mathrm{yr}\right.$ suffices) while wood and Cahn used $(1 / 4)$ the observed rates. That choice was forced by the 47 Tuc Miras plus overtone pulsation.

2) As I showed in Erice, Feast's temperature are completely consistent with my picture. However I feel that Teff is the most difficult parameter to determine observationally due to sphericity (which tends to give $\left.\mathrm{T}_{\text {obs }}<\mathrm{T}_{\text {eff }}\right)$. I therefore prefer to quote $\mathrm{T}_{\text {eff }}=3000 \mathrm{~K}( \pm 500$ ?). The luminosity is much more important in my formulation than is $\mathrm{T}_{\text {eff }}$. 